ORIGINAL ARTICLE

\title{
Antenatal steroids and fluid balance in very low birthweight infants
}

\author{
G Dimitriou, V Kavvadia, M Marcou, A Greenough
}

Arch Dis Child Fetal Neonatal Ed 2005;90:F509-F513. doi: 10.1136/adc.2005.071688

\begin{abstract}
See end of article for authors' affiliations

.....................

Correspondence to: Professor Greenough, Neonatal Intensive Care Centre, 4th Floor, Golden Jubilee Wing, King's College Hospital, London SE5 9RS, UK; anne. greenough@kcl.ac.uk
\end{abstract}

Accepted 2 May 2005

Published online first 5 May 2005

\begin{abstract}
Objectives: To determine if insensible water loss (IWL) differed between infants exposed or not exposed antenatally to corticosteroids and to explore possible mechanisms for the early postnatal diuresis associated with antenatal steroid exposure.

Design: Retrospective analysis of prospectively collected data.

Setting: Level three neonatal intensive care unit.

Patients: Ninety six infants, median gestational age 27.5 weeks (range 23-33).

Main outcome measures: Comparison of the IWL, urine output and osmolality, fluid input, electrolyte imbalance, respiratory illness severity (as assessed by surfactant requirement, maximum peak inspiratory pressure, and inspired oxygen concentration), and cardiovascular status (as assessed by inotrope requirement) between infants with antenatal corticosteroid exposure and gestational age matched controls.

Results: The infants exposed to antenatal steroids differed significantly from the controls in having both a lower IWL ( $p=0.0135)$ and a higher urine output $(p=0.0036)$ on day 1 , and fewer developed hyponatraemia $(p=0.027$ ) on day 2 . Fewer of those exposed to antenatal steroids required inotropes ( $p=0.06)$, but their respiratory status was similar to that of the controls.

Conclusions: Infants exposed to antenatal corticosteroids have a lower IWL. The results suggest that greater skin maturation, but also better perfusion rather than less severe respiratory status, explains the early diuresis in infants exposed to antenatal steroids.
\end{abstract}

$\mathrm{F}$ luid and electrolyte problems are very common in very prematurely born infants, partly because of their high insensible water loss (IWL) and partly because of high infusion rates of intravenous fluids which may be given in an attempt to compensate for the expected losses. Treatments that reduce IWL are therefore highly desirable. It has been suggested that corticosteroids administered antenatally may result in enhancement of fetal epithelial cell maturation and improvement in skin barrier function ${ }^{1}$ as, in one study, ${ }^{1}$ a higher early infant urine output after antenatal corticosteroid administration was associated with a lower IWL. In another study, ${ }^{2}$ however, no significant differences were seen in transepidermal water loss according to antenatal steroid status. It is clearly important to determine whether antenatal steroid exposure does reduce IWL, as this would inform decisions on appropriate fluid prescription. Thus our aim was to determine whether IWL differed significantly between infants with or without antenatal steroid exposure. Antenatal steroid administration potentially has many other effects on infants including improving their respiratory ${ }^{3}$ and cardiovascular status, ${ }^{4}$ which could affect urine output. Thus an additional aim was to explore possible mechanisms for the early postnatal diuresis associated with antenatal steroid exposure $^{1}$ by comparing fluid and electrolyte balance, inotrope requirement, and severity of respiratory illness in prematurely born infants exposed or not to antenatal steroids.

\section{METHODS}

A retrospective analysis was undertaken of prospectively collected data from very low birthweight infants born before 34 weeks gestation who had been entered in a randomised trial examining the effect of perinatal fluid input on the development of chronic lung disease. ${ }^{5}$ The study was approved by the research ethics committee of the King's
Healthcare NHS Trust. The infants had been randomised to receive one of two crystalloid fluid regimens regardless of their antenatal steroid exposure, but for the purposes of this paper they were then stratified according to steroid exposure status (see below). Fluid regimen A was $70 \mathrm{ml} / \mathrm{kg}$ on day $\mathrm{l}$, $90 \mathrm{ml} / \mathrm{kg}$ on day $2,110 \mathrm{ml} / \mathrm{kg}$ on day $3,120 \mathrm{ml} / \mathrm{kg}$ on day 4 , $140 \mathrm{ml} / \mathrm{kg}$ on day 5 , and $150 \mathrm{ml} / \mathrm{kg}$ on days 6 and 7 . Infants on fluid regimen $\mathrm{B}$, compared with those on regimen $\mathrm{A}$, were to be prescribed about $20 \%$ less maintenance fluid over the first week. Fluid input on either fluid regimen, however, was only increased if the infant had lost weight and there were no signs of fluid overload, such as hyponatraemia (serum sodium $<135 \mathrm{mmol} / \mathrm{l}$ ). An extra $30 \mathrm{ml} / \mathrm{kg} /$ day maintenance fluids was given while the infant received phototherapy. Phototherapy was started if the infant's bilirubin exceeded the phototherapy level for gestational age using standard charts. Colloid infusions were not routinely prescribed, but $5 \%$ albumin was administered to infants who were hypotensive but had no signs of heart failure, and fresh frozen plasma was given to infants with coagulation abnormalities. All fluid input (crystalloid and colloid) was recorded hourly and then totalled for the 24 hour period. Urine was collected on open nappies or, for extremely low birthweight babies, on cotton wool balls placed on the nappy. As soon as the infant voided, the nappy or cotton wool ball was weighed to determine the amount of urine passed. If an infant was oliguric and the bladder palpable, this was manually expressed. The urine output was then totalled for each 24 hour period. Infants were weighed at least daily using bedscales. From the daily fluid intake and output and weight, the IWL was estimated using the formula: IWL $=$ total fluid intake - fluid output + weight loss in grams.

Abbreviations: $\mathrm{FlO}_{2}$, fractional inspired oxygen concentration; GFR, glomerular filtrate rate; IWL, insensible water loss 
Table 1 Fluid balance in infants who were and were not exposed to antenatal steroids

\begin{tabular}{|c|c|c|c|}
\hline & Antenatal steroids & Controls & p Value \\
\hline Gestational age (weeks) & $27.5(23-33)$ & $27.5(23-33)$ & $>0.99$ \\
\hline Birth weight $(\mathrm{g})$ & $971(570-1500)$ & $892(600-1498)$ & 0.97 \\
\hline Caesarean section & $25(52)$ & $20(42)$ & 0.41 \\
\hline Female & $25(52)$ & $27(46)$ & 0.84 \\
\hline \multicolumn{4}{|l|}{ IWL (g) } \\
\hline Day 1 & $32(0-126)$ & $51(0-138)$ & 0.0135 \\
\hline Day 2 & $19(0-171)$ & $41(0-147)$ & 0.436 \\
\hline \multicolumn{4}{|l|}{ Fluid input $(\mathrm{ml} / \mathrm{kg})$} \\
\hline Day 1 & 77 (38-191) & $76(46-128)$ & 0.78 \\
\hline Day 2 & $89(39-146)$ & $86(35-143)$ & 0.56 \\
\hline \multicolumn{4}{|l|}{ Urine output $(\mathrm{ml} / \mathrm{kg})$} \\
\hline Day 1 & $38(4-105)$ & $22(1-117)$ & 0.0036 \\
\hline Day 2 & $89(26-204)$ & $80(0-148)$ & 0.264 \\
\hline \multicolumn{4}{|l|}{ Urine osmolality (mOsm/l) } \\
\hline Day 1 & 191 (103-1443) & $235(104-501)$ & 0.115 \\
\hline Day 2 & $221(104-501)$ & $234(76-484)$ & 0.88 \\
\hline \multicolumn{4}{|l|}{ Urine sodium (mmol/l) } \\
\hline Day 1 & 48 (9-139) & $56(16-199)$ & 0.081 \\
\hline Day 2 & $63(13-158)$ & $64(11-146)$ & 0.87 \\
\hline $\begin{array}{l}\text { Weight change by day } 2 \text { (\% of birth } \\
\text { weight) }\end{array}$ & $-1.05(-10.6$ to 20.4$)$ & $+1.18(-8.9$ to 17.8$)$ & 0.066 \\
\hline
\end{tabular}

All infants followed the unit's routine policy with regard to monitoring - that is, their urine osmolality was measured from the first urine passed each morning. Electrolytes, creatinine, and bilirubin were assessed daily, but more frequently if abnormal. Deviations from this policy occasionally occurred if an infant was stable and receiving more than half their nutrition enterally. No sodium supplements were given in the first 24 hours after birth, although the patency of indwelling arterial lines was maintained by an infusion of heparinised $0.45 \%$ saline. Subsequently the amount of sodium supplementation was altered to keep the serum sodium concentration between 135 and $145 \mathrm{mmol} / 1 .{ }^{6}$ Blood pressure was measured hourly, usually by intra-arterial monitoring. Hypotension (a mean blood pressure less than the infant's gestational age) was treated initially with up to two boluses of a colloid or crystalloid solution, and, if that failed to improve the blood pressure, a dopamine infusion was started. Inotropes were used as first choice for blood pressure support if there was evidence of myocardial ischaemia.

The infants were nursed in closed incubators with $75-80 \%$ humidification, except for the first few hours after birth when indwelling arterial and central lines were being placed. They were ventilated using humidified gases via oral shouldered endotracheal tubes, which we have previously shown to have minimal or no leak. ${ }^{7}$ Infants were started on enteral feeding $(0.5 \mathrm{ml} / \mathrm{h})$ as soon as their clinical condition permitted (the volume of enteral feeding was included in the crystalloid volume received). If the infant was intolerant of enteral feeds, parenteral feeding was prescribed from day four.

The nurses recorded each infant's ventilatory settings and inspired oxygen requirement on an hourly basis. Arterial blood gases were checked at least four hourly from samples obtained from catheters sited for clinical purposes. Ventilatory settings and the fractional inspired oxygen concentration $\left(\mathrm{FiO}_{2}\right)$ were adjusted to ensure the blood gases were kept in a pre-determined range $\left(\mathrm{pH} 7.25-7.40, \mathrm{PaO}_{2} 7-\right.$ $10 \mathrm{kPa}, \mathrm{PaCO}_{2} 5-7 \mathrm{kPa}$ ). From the nursing charts, the infant's maximum peak inspiratory pressure and $\mathrm{FIO}_{2}$ in the first 48 hours of birth were noted. Exogenous surfactant (Survanta) was administered to all infants with respiratory distress syndrome within two hours of birth if they were fully ventilated and had an inspired oxygen concentration of more than $30 \%$. A second dose was given 12 hours later if the infant was still fully ventilator dependent with a supplementary oxygen requirement. Infants were described as having a patent ductus arteriosus if the diagnosis had been confirmed by echocardiography and they had required treatment. All infants underwent serial cranial ultrasound examination at least in the first week after birth and were described as having an intracerebral haemorrhage if there was bleeding into the ventricles or brain substance.

Infants from the "fluid trial" ${ }^{5}$ whose mothers had received antenatal corticosteroid therapy within seven days of delivery were identified. The decision to administer antenatal steroids was by the obstetric staff on clinical grounds. The policy of the maternity unit was to administer corticosteroids (a complete course consisted of two doses of $12 \mathrm{mg}$ dexamethasone 12 hours apart) to all mothers in whom delivery was expected before 34 weeks gestation, unless the mother was septicaemic or delivery was imminent. An attempt was then made to match each subject by gestational age to an infant from the fluid trial whose mother had received no steroids antenatally (controls).

\section{Statistical analysis}

The data were tested using the Shapiro Wilk W and D'Agostino Skewness test and found to be non-normally distributed. Differences therefore between the two groups, in particular with regard to their IWL, urine output, severity of respiratory illness (as indicated by their maximum peak inspiratory pressure, and $\mathrm{FIO}_{2}$, and surfactant requirement), and cardiovascular status (as reflected by inotrope requirement), were assessed for statistical significance using the Wilcoxon rank sum test or $\chi^{2}$ test as appropriate.

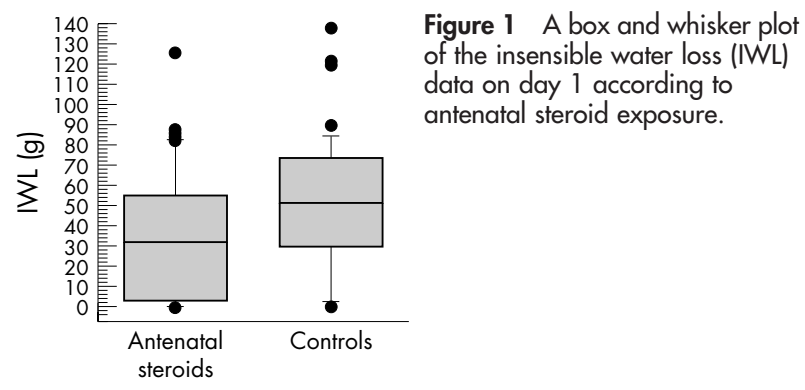




\begin{tabular}{|c|c|c|c|}
\hline & $\begin{array}{l}\text { Antenatal } \\
\text { steroids }\end{array}$ & Controls & p Value \\
\hline \multicolumn{4}{|l|}{ Day 1} \\
\hline Hyponatraemia & $6(16)$ & $7(18)$ & $>0.99$ \\
\hline Hypernatraemia & $2(5)$ & $5(13)$ & 0.432 \\
\hline Hypoglycaemia & $10(21)$ & $14(30)$ & 0.478 \\
\hline Phototherapy & $1(2)$ & $8(17)$ & 0.0305 \\
\hline \multicolumn{4}{|l|}{ Day 2} \\
\hline Hyponatraemia & $2(4)$ & $9(20)$ & 0.027 \\
\hline Hypernatraemia & $14(30)$ & $10(20)$ & 0.477 \\
\hline Hypoglycaemia & $13(30)$ & $12(26)$ & 0.815 \\
\hline Phototherapy & $9(20)$ & $16(34)$ & 0.160 \\
\hline
\end{tabular}

\section{RESULTS}

It was possible to match 48 infants whose mothers had received corticosteroids antenatally with 48 infants whose mothers had received no antenatal steroids. Twenty eight of the mothers had received a single complete course, nine had received more than two complete courses, and 11 a single dose of dexamethasone.

Infants whose mothers had received antenatal steroids had on day $\mathrm{l}$ a lower IWL ( $\mathrm{p}=0.0135$; table $\mathrm{l}$, fig $\mathrm{l}$ ) and also a higher urine output $(\mathrm{p}=0.0036)$. Fewer of the antenatal steroid group on day 1 required treatment with phototherapy $(\mathrm{p}=0.0305$; table 2$)$ or inotrope support $(\mathrm{p}=0.06$; table 3$)$ and on day 2 developed hyponatraemia $(\mathrm{p}=0.027)$. There were, however, no significant differences between the two groups with regard to their fluid input (table 1) or the severity of their respiratory illness (table 3).

\section{DISCUSSION}

We have shown that prematurely born infants who had been exposed to antenatal steroids have a lower IWL. This is in keeping with the findings of Omar et al, ${ }^{1}$ who reported lower IWL in each of the first four days after birth in 16 extremely low birthweight infants exposed to antenatal corticosteroids. Jain et $a l^{2}$ however, found no influence of either sex or antenatal steroids on transepidermal water loss from the abdominal skin of prematurely born infants. Their results are surprising given the effects of antenatal steroids in animal models. ${ }^{18-10}$ Female rat pups have been noted to have a more mature epithelial barrier than male pups of the same gestational age; on day 20 female rat pups had transepidermal water loss rates $40 \%$ less than that of male pups. ${ }^{10}$ The explanation for the sex differences in transepidermal water loss is that oestrogen accelerates, whereas testosterone retards, epidermal barrier maturation. Daily antenatal steroid administration in rats has been shown to accelerate the maturation rate of the stratum corneum and diminish transepidermal water loss. ${ }^{8}$ In addition, prematurely born rats exposed to antenatal steroids had accelerated epidermal/ peridermal maturation, resulting in less dehydrated skin. ${ }^{9}$ Jain et al ${ }^{2}$ compared infants exposed to antenatal steroids between 1997 and 1998 to historical controls born a decade earlier in 1985 to 1986. During that 10 year period, there have been many changes in neonatal care-for example, routine administration of exogenous surfactant; such changes may have biased their comparison between the two groups. ${ }^{2}$ In contrast, our groups were recruited contemporaneously and, although they must have differed with regard to the predictability of their premature delivery, they were individually matched for gestational age and were similar with respect to birth weight, mode of delivery, and routine aspects of neonatal care. In these two similar groups, we showed differences in IWL related to antenatal steroid exposure, which may be due to steroid induced maturation of the skin epithelial barrier. ${ }^{189}$

We confirmed that antenatal steroid administration is associated with a greater early urine output. ${ }^{1}$ This association may be explained by a specific effect of corticosteroids on renal function. Chronic treatment of methylprednisolone has been shown to increase the glomerular filtrate rate (GFR) of rats, ${ }^{11}$ and fetal lambs injected with betamethasone had a higher GFR than controls. ${ }^{11}$ In addition, antenatal steroids have been shown in both animal and human studies to affect renal cell differentiation, with an increase in renal adenylate cyclase activity and tubular function. ${ }^{12}$ Other studies, however, have not shown an effect of antenatal corticosteroids on either tubular function ${ }^{13}$ or GFR. ${ }^{13}$ Tubular reabsorption of $\beta_{2}$ microglobulin was not affected by prenatal glucocorticoid administration, and GFR (as estimated by the gentamicin half life) did not differ between infants exposed or not to one or more doses of betamethasone antenatally. ${ }^{13}$

The infants exposed antenatally to steroids had similar postnatal weight changes by day 2 (table 1). Thus, although they had a higher urine output on day 1 , this is likely to have been balanced by their significantly lower IWL. Indeed, their significantly lower requirement for phototherapy would suggest that they were not more dehydrated, and indeed on days 1 and 2 the proportion of infants with hypernatraemia did not differ significantly between the two groups. Hyponatraemia, however, was more common in the controls, suggesting that fluid balance was more appropriate in the infants exposed to antenatal steroids. It is possible that the higher incidence of hyponatraemia in the controls, given that they had a higher IWL and some had experienced weight

Table 3 Cardiovascular and respiratory status, morbidity, and mortality

\begin{tabular}{llll}
\hline & Antenatal steroids & Controls & p Value \\
\hline Inotrope support & $13(27)$ & $23(48)$ & 0.06 \\
$\quad$ Day 1 & $15(33)$ & $24(51)$ & 0.093 \\
Day 2 & $29(60)$ & $35(73)$ & 0.279 \\
Surfactant treatment & $18(14-38)$ & $20(12-42)$ & 0.412 \\
Max PIP $\left(\mathrm{cm} \mathrm{H}_{2} \mathrm{O}\right)$ & $54(21-100)$ & $64(21-100)$ & 0.148 \\
Max FIO & $9(19)$ & $16(33)$ & 0.163 \\
PDA & $5(10)$ & $9(19)$ & 0.368 \\
ICH & $25(64)$ & $22(59)$ & 0.814 \\
Oxygen dependency at: & $9(24)$ & $12(33)$ & 0.442 \\
28 days & $12(25)$ & $14(29)$ & 0.818 \\
36 weeks PCA & &
\end{tabular}

Data are number (\%) or median (range).

$\mathrm{PIP}$, Peak inspiratory pressure; $\mathrm{FIO}_{2}$, fractional inspired oxygen; PDA, patent ductus arteriosus; $\mathrm{ICH}$, intracerebral haemorrhage; PCA, postconceptional age. 


\section{What is already known on this topic}

- Fluid and electrolyte imbalance are very common in prematurely born infants, and this is partly due their high insensible water loss (IWL)

- Evidence on whether infants exposed antenatally to corticosteroids have a lower IWL is conflicting; they do, however, have an early postnatal diuresis, but whether this is explained by greater skin maturation and or other mechanisms remains uncertain

loss, may be explained by greater urine wasting of sodium. Overall, however, there was a small weight gain in the controls and thus the hyponatraemia may reflect fluid overload.

The group exposed antenatally to steroids were less likely to require inotrope support than the control group. This is in keeping with the finding that the mean arterial pressure was significantly higher in lambs treated antenatally with betamethasone. ${ }^{14}$ The exact mechanism for the blood pressure increases with antenatal glucocorticoid treatment are not clear. In lambs exposed antenatally to betamethasone, however, their cardiac output was unchanged, suggesting that their blood pressure increases may be related to increases in peripheral vascular resistance. The lower inotrope requirement of our infants exposed antenatally to corticosteroid may mean they had better renal perfusion and hence higher urine output. It is also possible that the higher urine output resulted from lower concentrations of arginine vasopressin reflecting better cardiovascular status. Postnatally, angiotensin II and arginine vasopressin concentrations have been shown to be significantly lower in betamethasone treated animals by about three hours after birth, ${ }^{15}$ and this was associated with an increase in urine output and sodium excretion. The lower arginine vasopressin concentrations were attributed to an improved cardiovascular status rather than steroid induced suppression of arginine vasopressin secretion.

Improvement in respiratory status is associated with a postnatal diuresis, ${ }^{16}{ }^{17}$ although the temporal relation of the two events is controversial. ${ }^{16}{ }^{17}$ Thus a possible explanation that we considered for the higher urine output of the antenatal steroid groups was that they may have milder respiratory disease, given the known effects of antenatal steroids. Antenatal steroid administration can improve respiratory function by earlier clearance of fetal lung fluid. ${ }^{18}$ Glucocorticoids have been shown to upregulate Na,K-ATPase mRNA expression in the immature mouse lung. ${ }^{18} \mathrm{Na}, \mathrm{K}-$ ATPase provides the energy for the active transport of sodium across the pulmonary epithelium and hence fetal lung fluid resorption. We, however, found no significant differences in the proportions of the two groups who required surfactant, nor in their maximum inspired oxygen or peak pressure requirement. We also did not find any significant differences in morbidity or mortality between our two groups, but it is possible that the numbers studied were too small to demonstrate an effect. Nevertheless, our results do not suggest that differences in respiratory status explain the higher urine output seen in the antenatal steroid group.

There are weaknesses to our study. The initial study was not designed to determine the effect of antenatal steroids on fluid balance and the infants were not randomised to receive antenatal steroids. The policy of the obstetricians was to administer corticosteroids to all mothers in whom delivery was expected before 34 weeks gestation, unless the mother

\section{What this study adds}

- This study shows that very prematurely born infants exposed antenatally to corticosteroids have lower IWL than gestational age matched controls

- The results suggest that greater skin maturation and better perfusion, rather than less severe respiratory status, explain the early diuresis in infants exposed to antenatal steroids

was septicaemic or delivery was imminent. Thus there are likely to be differences in the antenatal pathologies of the mothers of the two groups, with a greater incidence of such problems as premature rupture of the membranes and preeclampsia in the antenatal steroid group. We are, however, not aware that such pregnancy complications affect IWL in infants. Clearly, the best method of assessing the impact of antenatal steroids on fluid balance of very low birthweight infants would be to undertake a randomised trial, but, given the known benefits of antenatal steroids, such a trial would be unethical. We have therefore compared the results from infants exposed to antenatal steroids with controls matched for gestational age who were cared for contemporaneously, and thus our results are not biased by the infants being exposed to different management eras. A strength is that all the data collection was undertaken prospectively and was very detailed, allowing us to examine the effect of antenatal steroid exposure not only on fluid balance, but also blood pressure and respiratory status. Although most of the infants had been exposed to at least one complete course of antenatal steroids, a minority had only received one dose of dexamethasone. This may have lessened any effect of antenatal corticosteroid exposure, yet we still saw significant differences between our two groups.

We have shown that, compared with gestational age matched controls, prematurely born infants exposed antenatally to steroids had a lower IWL on day 1 . They also had a higher urine output, in association with a lower requirement for inotrope support, but no significant differences in their respiratory support. We therefore conclude that greater skin maturation and better renal perfusion explain the early diuresis noted in infants exposed to antenatal corticosteroids.

\section{ACKNOWLEDGEMENTS}

GD was supported by a Children Nationwide Medical Research Fund/ Nestle Research Fellowship, and VK by a project grant from the South East Thames NHS Research \& Development Directorate. We thank Mrs Deirdre Gibbons for secretarial assistance.

\section{Authors' affiliations}

G Dimitriou, V Kavvadia, M Marcou, A Greenough, Division of Asthma and Lung Biology, Guy's, King's and St Thomas' School of Medicine, King's College London, UK

Competing interests: none declared

\section{REFERENCES}

1 Omar SA, De Cristofaro JD, Agarwal BI, et al. Effects of prenatal steroids on water and sodium homeostasis in extremely low birthweight infants. Pediatrics 1999; 104:482-8.

2 Jain A, Rutter N, Cartlidge PHT. Influence of antenatal steroids and sex on maturation of the epidermal barrier in the preterm infant. Arch Dis Child Fetal Neonatal Ed 2000;83:F112-16.

3 Crowley P. Prophylactic corticosteroids for preterm birth. Cochrane Library, 2004, Issue 2.

4 Berry LM, Polk DH, Ikegami M, et al. Preterm newborn lamb renal and cardiovascular responses after fetal or maternal antenatal betamethasone. Am J Physiol 1999;272:1972-9. 
5 Kavvadia V, Greenough A, Dimitriou G, et al. Randomised trial of fluid restriction in ventilated very low birthweight infants. Arch Dis Child Fetal Neonatal Ed 2000;83:91-6.

6 Shaffer SG, Weismann DN. Fluid requirements in the preterm infant. Clin Perinatol 1992;19:233-50.

7 Hird M, Greenough A, Gamsu H. Gas trapping during high frequency positive pressure ventilation using conventional ventilators. Early Hum Dev 1990;22:51-6

8 Aszterbaum M, Feingold KR, Menon GK, et al. Glucocorticoids accelerate fetal maturation of the epidermal permeability barrier in the rat. $J$ Clin Invest 1993;91:2703-8

9 Okah FA, Pickens WL, Hoath SB. Effect of prenatal steroids on skin surface hydrophobicity in the premature rat. Pediatr Res 1995:37:402-8.

10 Hanley K, Rassner U, Jiang Y, et al. Hormonal basis for the gender difference in epidermal barrier formation in the fetal rat. Acceleration by oestrogen and delay by testosterone. J Clin Invest 1996;97:2576-84.

11 Baylis C, Brenner BM. Mechanisms of the glucocorticoid-induced increase in glomerular filtration rate. Am J Physiol 1978;234:F166-70.
12 Slotkin TA, Seidler FJ, Kavlock RJ, et al. Fetal dexamethasone exposure accelerates development of renal function: relationship to dose, cell differentiation and growth inhibition. J Dev Physiol 1992;17:55-61.

13 MacKintosh D, Baird-Lambert J, Drage D, et al. Effects of prenatal glucocorticoids on renal maturation in newborn infants. Dev Pharmacol Ther 1985;8:107-14.

14 Smith LM, Ervin G, Wada N, et al. Antenatal glucorticoids after postnatal preterm lamb renal and cardiovascular responses to intravascular volume expansion. Pediatr Res 2000:47:622-7.

15 Ervin MG, Berry LM, Ikegami M, et al. Single dose fetal betamethasone administration stabilizes postnatal glomerular filtration rate and alters endocrine function in premature lambs. Pediatr Res 1996;40:645-51.

16 Heaf DP, Belik J, Spitzer AR, et al. Changes in pulmonary function during the diuretic phase of respiratory distress syndrome. J Pediatr 1982:101:103-7.

17 Kavvadia V, Greenough A, Dimitriou G, et al. Comparison of respiratory function and fluid balance in very low birthweight infants given artificial or natural surfactant or no surfactant treatment. J Perinat Med 1998;26:469-74.

18 Celsi G, Wang ZM, Akusjarvi G, et al. Sensitive periods of glucocorticoids' regulation of $\mathrm{Na}^{+}, \mathrm{K}^{+}$-ATPase mRNA in the developing lung and kidney. Pediatr Res 1993;33:5-9.

\section{bmjupdates+}

bmjupdates+ is a unique and free alerting service, designed to keep you up to date with the medical literature that is truly important to your practice.

bmjupdates+ will alert you to important new research and will provide you with the best new evidence concerning important advances in health care, tailored to your medical interests and time demands.

\section{Where does the information come from?}

bmjupdates+ applies an expert critical appraisal filter to over 100 top medical journals A panel of over 2000 physicians find the few 'must read' studies for each area of clinical interest

Sign up to receive your tailored email alerts, searching access and more...

www.bmjupdates.com 